\title{
A Method for Robust Time-Reversal Focusing in a Fluctuating Ocean
}

\author{
Seongil Kim, W. A. Kuperman, W. S. Hodgkiss, H. C. Song, G. F. Edelmann \\ Scripps Institution of Oceanography, La Jolla, CA 92093-0238 \\ T. Akal \\ SACLANT Undersea Research Centre, 19138 La Spezia, Italy \\ R. P. Millane \\ Purdue University, West Lafayette, IN 47097-1160 \\ D. Di Iorio \\ University of Georgia, Athens, GA 30602-3636
}

\begin{abstract}
In recent years, we have demonstrated time-reversal mirrors (TRM) in the ocean. A focus of up to $30 \mathrm{~km}$ was achieved with low frequency $(445 \mathrm{~Hz})$ transmissions and the focal structure could be maintained over several days at a range of $15 \mathrm{~km}$. However, the stable focus was limited to less than an hour with high frequency $(3.5 \mathrm{kHz})$ transmissions due to the sensitive response of high frequency sound propagation to the medium fluctuations. In this study, an approach for robust time-reversal focusing is investigated based on a method developed for matched-field processing. Instead of using a single probe source pulse, the method makes use of several probe source pulses obtained over a certain period of time where each ping represents a different propagation condition of the medium. The back-propagation from a TRM weighted by a linear combination of the dominant singular vectors obtained from the signal matrix leads to stable focusing for a longer period of time than that with a single probe pulse. The proposed method is useful in non-static propagation conditions and when frequent probe signals are not available.
\end{abstract}

\section{INTRODUCTION}

Time reversal [1-3] exploits the time-reversal invariance of the wave equation in a static medium such that the retransmitted waveform from a time-reversal mirror (TRM) propagates backward to the position of probe source (PS) where the signal is generated. Since time-reversal focusing utilizes the reciprocity of a static medium, its performance can be degraded in a non-static environment where the propagation conditions change during the time of forward and backward propagation between the TRM and PS. In recent experiments the stability of the focal structure was investigated by repeatedly re-transmitting a stored probe source pulse into the time-dependent ocean medium. Measurements with high frequency $(3.5 \mathrm{kHz})$ transmissions showed that the stable focus was limited to an hour or a few minutes depending on the depth of the PS and the strength of the ocean variability implying that the effect of medium fluctuation is not trivial for such high frequency transmissions.

In this study, a method for robust time-reversal focusing is investigated based on Krolik's MV-EPC processor [4] originally developed for matched-field processing. Instead of using a single probe source pulse, the method makes use of several PS pings obtained over a time interval where each ping represents a different propagation condition of the ocean medium. The back-propagated field weighted by a linear combination of the singular vectors corresponding to the dominant singular values of the received signals produces stable focusing for a longer period of time than that with a single probe pulse. In the next section, experiment results are briefly described. In Sec. III, time-reversal focusing in a random ocean channel is addressed using adiabatic normal mode theory. In Sec. IV, the proposed method for robust time-reversal focusing is described. Simulation results are discussed in Sec. V.

\section{TIME-REVERSAL EXPERIMENTS}

\section{A. Experimental setup}

A series of time reversal experiments was performed in two areas, near Formiche and Elba islands, off the west coast of Italy depicted as shaded boxes in Fig. 1. Both areas are relatively flat environments with water depth of about $130 \mathrm{~m}$ in the Formiche area and $120 \mathrm{~m}$ in the Elba area, respectively. Sound-speed profiles (SSP) were measured frequently by conductivity-temperature-depth (CTD) casts. The SSPs collected over the experiment period indicate the variability of upper water column especially in the thermocline region.

Fig. 2 shows the components of a TRM experiment. In the April 1996 and May 1997 experiments, a TRM was deployed near Formiche di Grosseto. The TRM spanned $77 \mathrm{~m}$ of the water column with 20 receiving hydrophones and 20 contiguously located slotted cylinder sources. Each element of the TRM was operated at a nominal source level of $160 \mathrm{~dB}$ re $1 \mathrm{uPa}$ with a center frequency of $445 \mathrm{~Hz}$. A PS was located in the vicinity of a 48 element vertical receive array (VRA). The July 1999 experiment was performed with a center frequency of $3.5 \mathrm{kHz}$ in both the Formiche and Elba areas and the May 2000 experiment in the Elba area. The experimental setup was similar to the lower frequency experiments but the TRM had 29 transducers spanning a 78$m$ aperture with a nominal source level of $178 \mathrm{~dB}$ re $1 \mathrm{uPa}$. The VRA covered $93 \mathrm{~m}$ of the water column with 32 hydrophones.

\section{B. Measured time-reversal stability}

If the ocean is static, PS pulses can be stored ahead of time to focus at specific locations. However, the temporal 


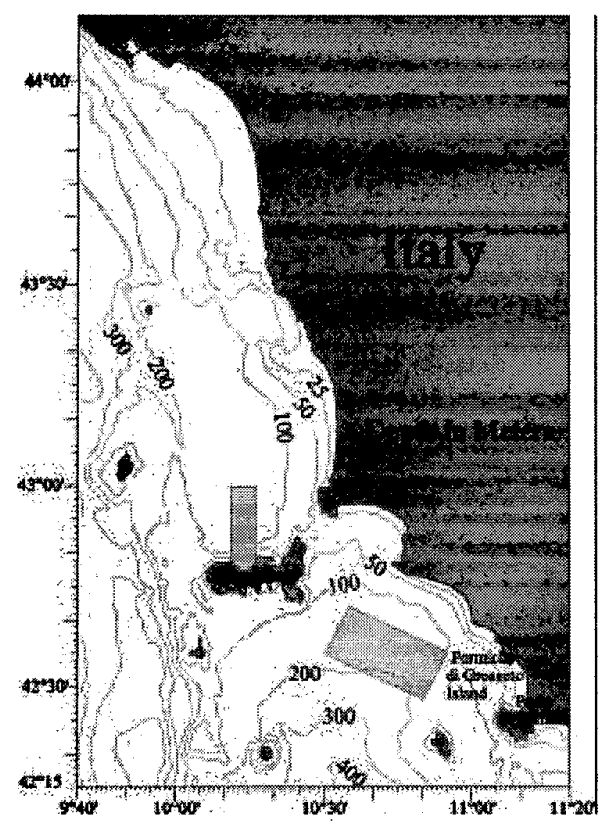

Fig 1. Locations of the time-reversal experiments. Low frequency ( 445 $\mathrm{Hz}$ ) experiments were performed near the Formiche di Grosseto and high frequency $(3.5 \mathrm{kHz})$ experiments were carried out both in the Elba and Formiche area

variability of the ocean is expected to limit such a procedure. In the May 1997 experiment, we found that probe pulses of $445 \mathrm{~Hz}$ still produced a significant focus at the original probe-source location up to 1 week later [5]. But recent experiments with $3.5 \mathrm{kHz}$ transmissions showed that a stable focus was severely limited by the medium fluctuations.

Fig. 3 shows the measured time-reversed pulses received on the VRA by re-transmitting the received probe pulse after a short time (16 min), 1 day, and 1 week later, respectively. The range between the TRM and PS was $15 \mathrm{~km}$ with the PS depth at $81 \mathrm{~m}$. The PS signal was a $50-\mathrm{ms}$ pure tone pulse at $445 \mathrm{~Hz}$. During the period, the focus moved a few meters downward resulting from the changes of modal shapes due to the increased sound speed. Although the focus is significantly degraded after a week along with the appearance of a sidelobe in the upper water column, the TRM clearly retained a focus.

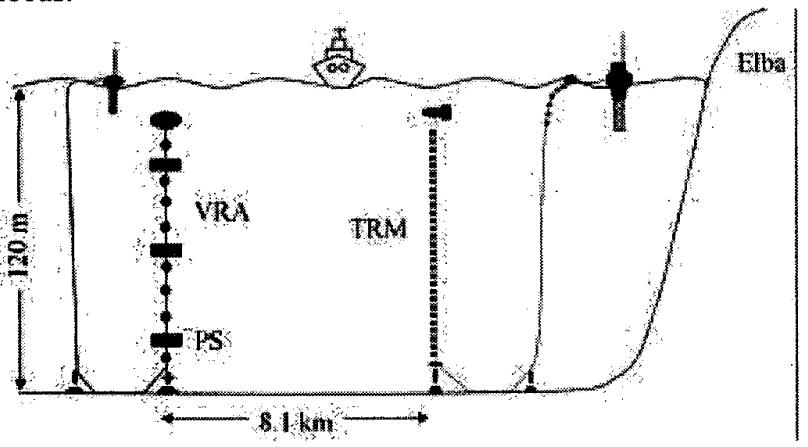

Fig. 2. Experimental setup for the high frequency $(3.5 \mathrm{kHz})$ timereversal experiment. The time-reversal mirror (vertical source-receiver array) consisted of 29 transducers with inter-element spacing $2.786 \mathrm{~m}$ spanning 78 $\mathrm{m}$ of the water column.

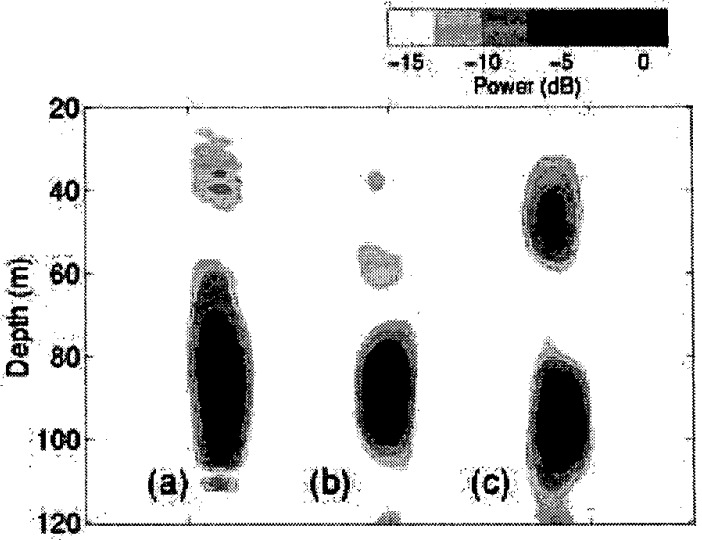

Fig. 3. Measured time-reversal foci with 50 -ms low frequency $(445 \mathrm{~Hz})$ transmissions. A $50-\mathrm{ms}$ pulse received by a TRM from a PS $15.2 \mathrm{~km}$ away at a depth of $81 \mathrm{~m}$ was re-transmitted (a) $16 \mathrm{~min}$, (b) 1 day, and (c) 1 week later.

Fig. 4 shows the measured high frequency time-reversal foci obtained by re-transmitting the probe-source pulse every minute over $45 \mathrm{~min}$. The pulse was $10-\mathrm{ms}$ long. The probe source was $8.1 \mathrm{~km}$ away from the TRM at the depth of $68 \mathrm{~m}$. Although the focal size does not change very much, the peak level of the focus decreases about $5 \mathrm{~dB}$ over the $45 \mathrm{~min}$. The focal structure shows slight upward movement between 15 and $30 \mathrm{~min}$ and the corresponding strong side-lobes appear at a depth around $75 \mathrm{~m}$.

Fig. 5 shows the results of a stability test made at different source depths. The TRM transmitted $100-\mathrm{ms}, 3-4 \mathrm{kHz}$ chirp signals every minute for $35 \mathrm{~min}$ by exciting the transducers one by one at a $333-\mathrm{ms}$ interval. The signals received by one element of the VRA are data sets of dimension $29 \times 35$, corresponding to the number of elements in the TRM and the number of transmission sequences. The first 29 data are correlated with successive data sets to examine the time variation of the signal correlation. This is a passive timereversal (phase-conjugation) process [6] performed using one-way transmission data. The result with all the receiver depths shows the vertical structure of the time-reversal stability. As we see the focus exits longer at deeper depths below the thermocline where the sound-speed fluctuations are expected to be small. The stability decreases with the probe source moving to shallow depths and shows minimum stability at a depth of $50 \mathrm{~m}$ which corresponds to the bottom of the thermocline.

\section{TIME-REVERSAL FOCUSING IN A RANDOM OCEAN}

In this section, we examine time-reversal focusing in a time-dependent ocean waveguide using adiabatic normal mode theory and a first-order perturbation approximation.

The time-reversal process consists of exciting a TRM by the phase conjugated received signals. Let the acoustic field at frequency $\omega$ at the $j$ th element of the TRM due to a probe source located at a horizontal distance $R$ and depth $z_{s}$ be 


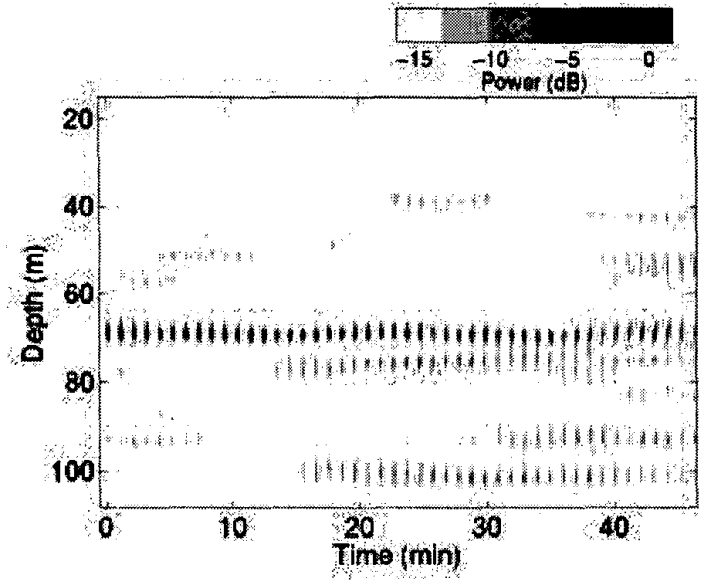

Fig. 4. Measured time-reversal foci with high frequency $(3.5 \mathrm{kHz})$ transmissions. A stored $10-\mathrm{ms}$ probe pulse received by the TRM from a PS at a range of $8.1 \mathrm{~km}$ was re-transmitted repeatedly for $45 \mathrm{~min}$.

$G\left(\mathbf{r}_{j}, \mathbf{r}_{s}\right)$, where $\mathbf{r}_{j}=\left(0, z_{j}\right)$ and $\mathbf{r}_{s}=\left(R, z_{s}\right)$. Then, the pressure field observed at a field point $\mathbf{r}=(r, z)$ reduces to a sum of time-reversal processes over the TRM transducer positions,

$$
p(\mathbf{r})=\sum_{j} G_{t 2}\left(\mathbf{r}, \mathbf{r}_{j}\right) G_{t 1}^{*}\left(\mathbf{r}_{j}, \mathbf{r}_{s}\right)
$$

where [ $]^{*}$ is complex conjugation. Specification of $t 1$ and $t 2$ allows for a time delay in the two-way propagation between the forward probe signal propagation and the backpropagation from the TRM. Here we assume that environmental changes during the short time interval of the one-way propagation between the PS and TRM are negligible. Using adiabatic normal mode theory, the acoustic field at a field point is

$$
\begin{array}{r}
p(\mathbf{r}, \Delta t) \approx \sum_{n} \sum_{m} \sum_{j} \frac{u_{m}(\mathbf{r}) u_{m}\left(\mathbf{r}_{j}\right) u_{n}\left(\mathbf{r}_{j}\right) u_{n}\left(\mathbf{r}_{s}\right)}{\rho\left(z_{s}\right) \rho(z) \sqrt{k_{n}^{*} k_{m} r R}} \\
\times \exp i\left(\int_{0}^{r} k_{m} d r^{\prime}-\int_{0}^{R} k_{n}^{*} d r\right)
\end{array}
$$

where $\Delta t=t 2-t l$ is the elapsed time of the time-reversal process and the subscript $\mathrm{n}$ and $\mathrm{m}$ are associated with the variables at time $t 1$ and $t 2$, respectively. If a TRM adequately samples most of the modes and the modal functions do not change very much during $\Delta t$, according to the orthonormality condition of modes, Eq. (2) becomes

$$
p(\mathbf{r}, \Delta t) \approx \sum_{n} \frac{u_{n}(\mathbf{r}) u_{n}\left(\mathbf{r}_{s}\right)}{\rho\left(z_{s}\right) k_{n} \sqrt{r R}} \exp i\left[k_{n}^{(\theta)}(r-R)+\Delta \theta_{n}\right]
$$

Using first-order perturbation theory, the perturbed horizontal

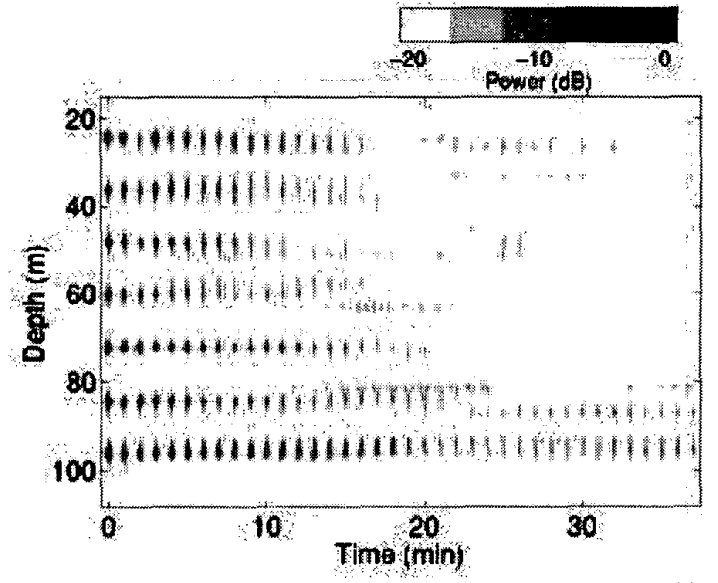

Fig. 5. High frequency stability test with $100-\mathrm{ms}, 3-4 \mathrm{kHz}$ chirp signals. A TRM transmitted the pulse element by element for $35 \mathrm{~min}$. The received signals at the VRA were correlated with the initial arrivals. The result shows different stability with different probe-source depths.

wavenumber due to small changes in sound speed $\delta c(r, z)$ from the background sound field $c_{0}(z)$ is approximated as $k_{n}(r, t) \cong k_{n}^{(0)}+\delta k_{n}(r, t)$. The modal phase perturbation from the two-way time-reversal propagation is $\Delta \theta_{n}=\theta_{n}(t 2)-\theta_{n}(t I)$ where $\theta_{n}=\int \delta k_{n} d r$. At the range of the probe source position, $r=R$, the closure property of the modes, $\sum_{n} u_{n}(z) u_{n}\left(z_{s}\right) / \rho(z) \equiv \delta\left(z-z_{s}\right)$, implies the focus is at the depth $z=z_{s}$. However, modal attenuation and the modal phase perturbation degrade the focus as

$p(R, z) \approx \sum_{n} \frac{u_{n}(z) u_{n}\left(z_{s}\right)}{\rho\left(z_{s}\right) k_{n} R} \exp \left(-2 \alpha_{n} R\right) \exp \left(\mathrm{i} \Delta \theta_{n}\right)$

where $\alpha_{n}$ is the imaginary part of $k_{n}^{(0)}$. Modal attenuation increases the focal size with increasing range due to the mode stripping of the higher order modes. Although different modes would have different $\Delta \theta_{n}$, these values can be highly correlated with each other due to the fact that the number of effective modes of the ocean fluctuations is normally much smaller than that of acoustic modes. This correlated $\Delta \theta_{n}$ between modes results in an environment-driven focal shift which is observed in the experimental data as the in-and-out of focal structure and is similar to the focal shift with a frequency change [7].

\section{ROBUST FOCUSING WITH SINGULAR VECTORS}

Given the model of Eq. (3), a direct approach for reducing the sensitivity of the time-reversal process to sound-speed variations is to minimize the effect of $\exp \left(i \Delta \theta_{n}\right)$ by modifying the received signal to a form suitable for the environment of back-propagation. Without having exact $a$ priori environmental information along the propagation path, 

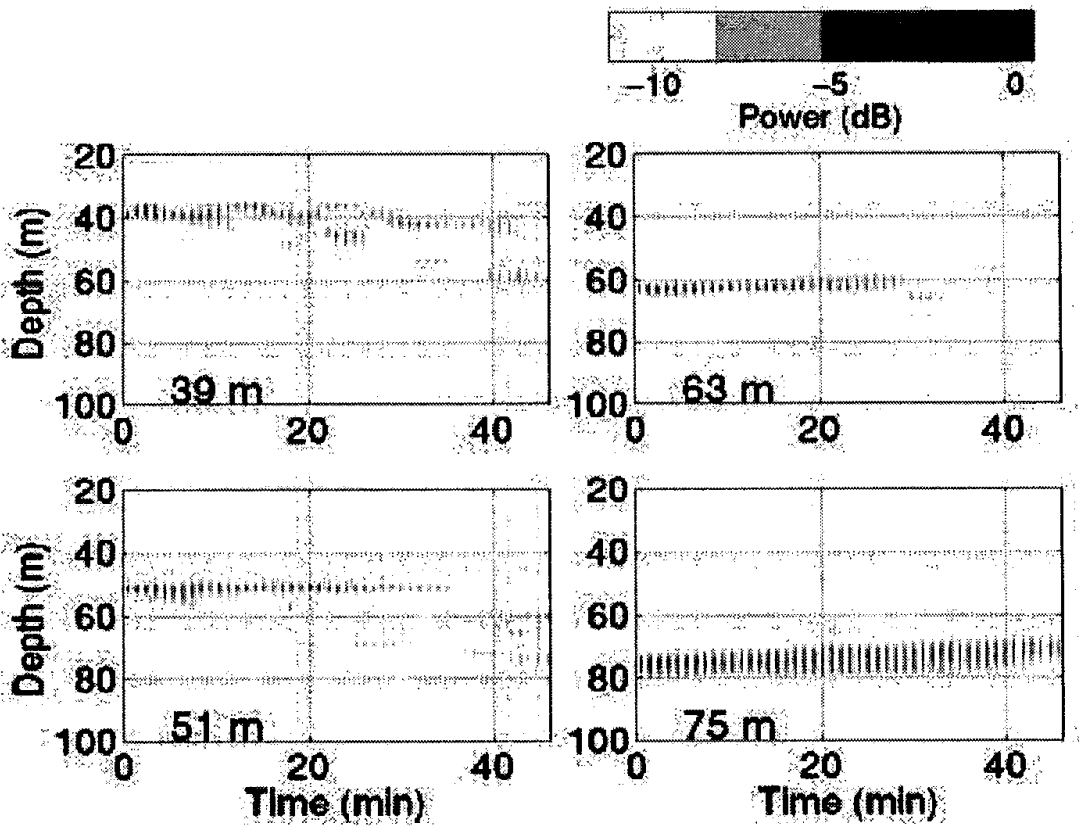

Fig. 6. Simulated time-reversal foci at 4 different probe-source depths. The pulse is a $100 \mathrm{~ms}$ pure tone at $3.5 \mathrm{kHz}$ and the range between the TRM and PS is $8 \mathrm{~km}$.

one possible method is using weight vectors that span the range of possible wave-front perturbations. By backpropagating multiple wave fronts we can minimize the phase perturbation and avoid the case of extreme mismatch between forward and backward propagation. This method [4] was originally developed for matched-field processing to improve the robustness to environmental mismatch. In matched-field processing, the possible wave-front perturbations for a particular geographical region are calculated from historical databases of sound-speed profiles. Similarly, in time-reversal processing we use multiple probesource pings obtained over a period of time where each ping represents a different propagation condition of the ocean medium.

If we measure successive PS pings, the number of data sets spanning all possible wave-front perturbations can be large. But this does not mean the effective dimension of the perturbation space is also large. The modal phase perturbation can be highly correlated among the modes as well as signal pings. The design of an efficient constraint space for the weight vector consists of selecting minimum vectors that can best approximate the phase perturbation space. This order reduction can be achieved using a singular value decomposition of the signal matrix $\mathbf{P}$ with rank $K$ approximation,

$$
\mathbf{P}=\mathbf{U D V}^{+}
$$

where []$^{+}$is the Hermitian transpose, $\mathrm{U}$ is $J \times K$ matrix whose columns are left singular vectors, $\mathbf{D}$ is $K \times K$ matrix whose diagonal elements are singular values of $\mathbf{P}$ and $\mathbf{V}$ is $N \times K$ matrix whose columns are right singular vectors. Here $J$ is the number of receivers in TRM and $N$ is the number of probe-source pings observed. Now the weighting vector $H$ for back-propagation is obtained by the linear combination of left singular vectors,

$$
\mathbf{H}=\mathbf{U q}
$$

where $\mathbf{q}$ is a $K \times 1$ vector representing the coefficient used for the linear combination of the singular vectors. Since the singular values decrease rapidly with increasing index number, in many cases the first singular vector is sufficient for stable focusing, which gives $q=[1,0 \ldots 0]^{T}$. Substituting $G_{t 1}$ in Eq. (1) with $\mathbf{H}$ in Eq. (6), the focused acoustic field becomes

$$
p(\mathbf{r})=\sum_{j} G_{t 2}\left(\mathbf{r}, \mathbf{r}_{j}\right) H^{*}\left(\mathbf{r}_{j}, \mathbf{r}_{s}\right)
$$

Here $p(\mathbf{r})$ should show a stable focal structure since the weight vector $H\left(\mathbf{r}_{j}, \mathbf{r}_{s}\right)$ maintains high correlation with $G_{t 2}$ for all possible wave-front perturbations. The singular value decomposition of the received signal vectors makes the focal size larger resulting in a stable focus under a slight shifting of the focal center caused by the perturbed modal phases.

\section{NUMERICAL SIMULATIONS}

In this section, numerical simulation results for the proposed method are described. The first step is to generate the time series of sound-speed perturbations. A major source 

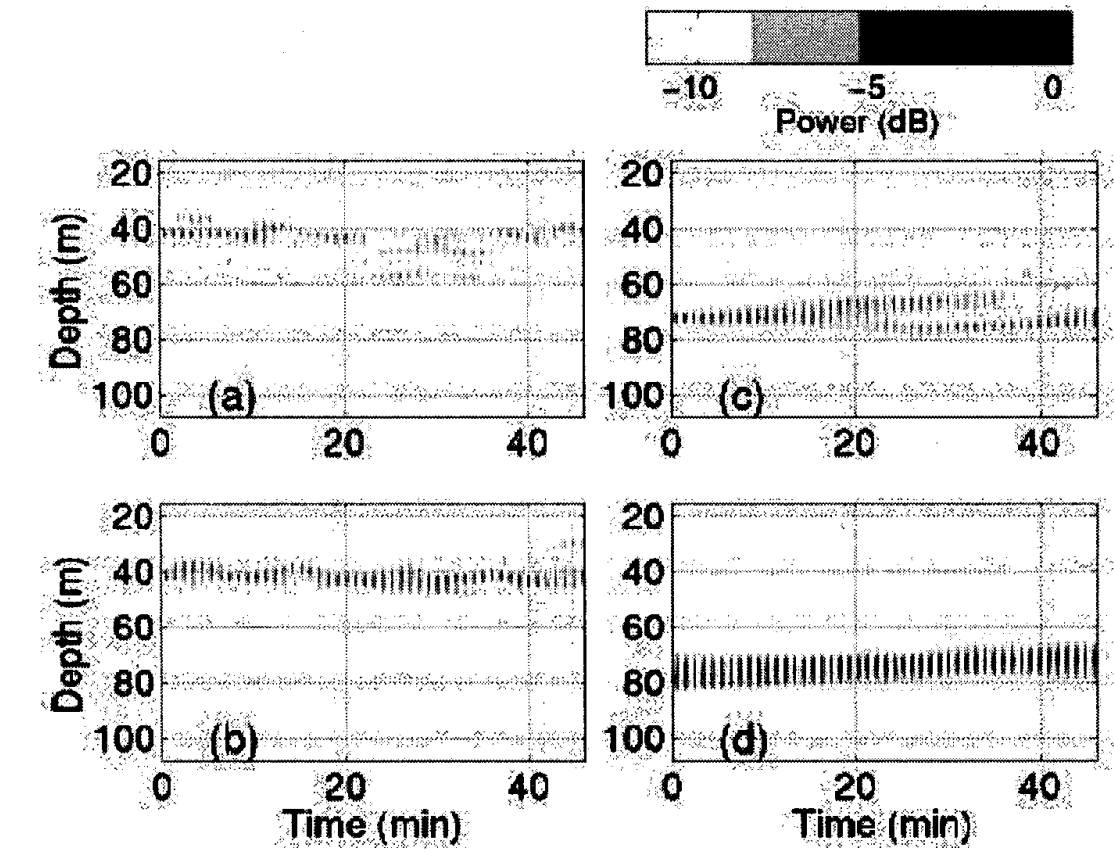

Fig. 7. Simulated time-reversal foci with 2 different probe source depths (a) and (b) at $42 \mathrm{~m}$ and (c) and (d) at $72 \mathrm{~m}$. Upper panels (a) and (c) correspond to using a single probe signal and lower panels (b) and (d) correspond to using the dominant singular vector.

of sound-speed fluctuations for ranges on the order of $10-\mathrm{km}$ is internal waves. The internal wave generation parameters were chosen to simulate the environmental conditions of the May 2000 experiment performed near Elba Island. The representative buoyancy frequency profile is determined by the average value of the CTD casts obtained during the experiment. We used the Garret-Munk internal wave spectrum with modified parameters suitable for shallow water (see Yang and Yoo, 1999 [8] for detäils).

A parabolic equation model was used to calculate the acoustic field between the TRM and the VRA for each time frame (1-min interval) of the sound-speed evolution. The TRM consists of 29 elements spanning water column from 30 to $107 \mathrm{~m}$ with $2.75-\mathrm{m}$ inter-element spacing. The VRA has 32 elements covering $93 \mathrm{~m}$ of the water column from 15 to $108 \mathrm{~m}$. Model parameters are selected from the waveguide condition of the Elba area.

Fig. 6 shows the simulated time-reversal foci for 4 different probe-source depths. The probe pulse is $100 \mathrm{~ms}$ long at $3.5 \mathrm{kHz}$. The calculated TRM signal was retransmitted into the simulated time-dependent medium every min for $45 \mathrm{~min}$. As expected the result shows increased stability at deeper depth below the thermocline zone. For the probe source at $39 \mathrm{~m}$, it appears that high frequency components of internal waves limit the stable focus to within $10 \mathrm{~min}$. At the $51 \mathrm{~m}$ and $63 \mathrm{~m}$ depths, the focus exists for about $20 \mathrm{~min}$. Even for the focus at a depth of $75 \mathrm{~m}$, which is in the relatively stable portion of the water column, we can identify a slow upward motion of the focus due to internal waves. The result matches well with the general tendency of the stability observed from the experiment.
In order to test the proposed method, the singular value decomposition approach was applied to the simulated probesource signals. We used 30 pings received for an hour and the analysis was performed at a center frequency $3.5 \mathrm{kHz}$. Fig. 7 shows the results from using the proposed method. The upper panel shows the time-reversal foci with a single probe signal transmitted repeatedly for $45 \mathrm{~min}$. The lower panel shows the results from transmitting the signal using the first dominant singular vector as data for the TRM. At $42 \mathrm{~m}$, the back-propagation with a single probe signal maintains a good focus for the first few minutes, then the focus begins fluctuating after $10 \mathrm{~min}$ and the focus is lost two times between 20 to $40 \mathrm{~min}$. In contrast, the back-propagation using the proposed method maintains the focus for the entire time interval. For the 72-m case, the focus is separated into two parts after $20 \mathrm{~min}$ for $15 \mathrm{~min}$ but the proposed method maintains the focus at a single spot. Although the focal size becomes larger, the proposed method maintains the focus longer showing the improved stability.

\section{SUMMARY}

The results from recent time-reversal experiments show that for high frequency transmissions $(3.5 \mathrm{kHz})$ stable focusing is severely limited due to the time-dependent soundspeed structures. A method for robust time-reversal focusing is proposed and tested through numerical simulations. The method utilizes multiple probe signals to extract singular vectors. Focusing using the dominant singular vector shows improved robustness of time-reversal focusing in a timedependent ocean. 


\section{ACKNOWLEDGMENTS}

This research was supported by the Office of Naval Research, Grant No. N00014-94-1-0458.

\section{REFERENCES}

[1] M. Fink, C. Prada, F. Wu, and D. Cassereau, "Self focusing with time reversal mirror in inhomogeneous media," Proceeding of IEEE Ultrasonics Symposium 1989 Montreal, pp. 681-686, 1989.

[2] D. R. Jackson and D. R. Dowling, "Phase conjugation in underwater acoustics," J. Acoust. Soc. Am., V89, pp. 171-181, 1991.

[3] W. A. Kuperman, W. S. Hodgkiss, H. C. Song, T. Akal, C. Ferla and D. Jackson, "Phase conjugation in the ocean: Experimental demonstration of an acoustic timereversal mirror," J. Acoust. Soc. Am., V102, pp. 25-40, 1998.

[4] J. L. Krolik, "Matched-field minimum variance beamforming in a random ocean channel," J. Acoust. Soc. Am.", V92, pp. 1408-1419, 1992.

[5] W. S. Hodgkiss, H. C. Song, W. A. Kuperman, T. Akal, C. Ferla and D. R. Jackson, "A long-range and variable focus phase-conjugation experiment in shallow water," J. Acoust. Soc. Am., V105, pp. 1597-1604, 1999.

[6] D. R. Dowling, "Acoustic pulse compression using passive phase-conjugate processing," J. Acoust. Soc. Am., V95, pp. 1450-1458, 1994.

[7] H. C. Song, W. A. Kuperman, and W. S. Hodgkiss, "A time-reversal mirror with variable range focusing," $J$. Acoust. Soc. Am., V103, pp. 3234-3240, 1998.

[8] T. C. Yang and K. Yoo, "Internal wave spectrum in shallow water: Measurement and comparison with the Garrett-Munk model," IEEE J. Ocean. Eng., V24, pp. 333-345, 1999 